\title{
磁光透明陶瓷的研究进展
}

\author{
李 江 $^{1}$, 戴佳卫 ${ }^{1}$, 潘裕柏 ${ }^{2}$
}

(1. 中国科学院 上海硅酸盐研究所, 透明光功能无机材料重点实验室, 上海 $200050 ; 2$. 上海师范大学 物理学院, 上海 200234)

摘 要: 磁光材料是指从紫外到红外波段具有磁光效应的光功能材料, 按照材料的类型可将其分为磁光玻璃、磁光 晶体、磁光透明陶瓷等。其中, 磁光透明陶瓷是近年来出现的一种新型磁光介质材料, 具有高 Verdet 常数、大尺寸、 高热导率、高激光损伤阈值等优点, 因而是用于高功率激光器中法拉第隔离器最理想的材料之一。目前已经报道的 磁光透明陶瓷材料主要包括琙镓石榴石 $\left(\mathrm{Tb}_{3} \mathrm{Ga}_{5} \mathrm{O}_{12}, \mathrm{TGG}\right)$ 陶瓷、铽铝石榴石 $\left(\mathrm{Tb}_{3} \mathrm{Al}_{5} \mathrm{O}_{12}, \mathrm{TAG}\right)$ 陶瓷以及一些倍半氧 化物陶瓷, 如氧化铽 $\left(\mathrm{Tb}_{2} \mathrm{O}_{3}\right)$ 陶瓷、氧化钬 $\left(\mathrm{Ho}_{2} \mathrm{O}_{3}\right)$ 陶瓷、氧化镝 $\left(\mathrm{Dy}_{2} \mathrm{O}_{3}\right)$ 陶瓷等。本文首先介绍了几种常见的磁光效 应，详细阐述了法拉第效应和克尔效应的基本原理。着重对几种磁光透明陶瓷材料的研究进展、材料性能、应用前 景进行了综述和介绍, 并对这几种磁光透明陶瓷的性能进行了比较和分析, 指出了它们存在的问题和今后的研究 方向。

关 键 词: 磁光透明陶瓷; 法拉第隔离器; 研究进展; 展望; 综述

中图分类号: TQ174 文献标识码: A

\section{Research Progress on Magneto-optical Transparent Ceramics}

\author{
LI Jiang ${ }^{1}$, DAI Jia-Wei ${ }^{1}$, PAN Yu-Bai ${ }^{2}$
}

(1. Key Laboratory of Transparent Opto-Functional Inorganic Materials, Shanghai Institute of Ceramics, Chinese Academy of Sciences, Shanghai 200050, China; 2. Department of Physics, Shanghai Normal University, Shanghai 200234, China)

\begin{abstract}
Magneto-optical material is a kind of optical functional material which has the magneto-optical effect from ultraviolet to infrared band. According to the type of material, magneto-optical materials can be classified into magneto-optical glass, magneto-optical crystals, magneto-optical transparent ceramics, etc. As a new type of magnetooptical material which has emerged in recent years, magneto-optical transparent ceramics are considered as one of the most promising candidates for Faraday isolators used in high power lasers due to its high Verdet constant, large size, high thermal conductivity, and high laser-induced-damage threshold. Up to now, the magneto-optical transparent ceramics reported mainly include terbium gallium garnet $\left(\mathrm{Tb}_{3} \mathrm{Ga}_{5} \mathrm{O}_{12}, \mathrm{TGG}\right)$, terbium aluminum garnet $\left(\mathrm{Tb}_{3} \mathrm{Al}_{5} \mathrm{O}_{12}, \mathrm{TAG}\right)$ and some sesquioxide ceramics such as terbium oxide $\left(\mathrm{Tb}_{2} \mathrm{O}_{3}\right)$, holmium oxide $\left(\mathrm{Ho}_{2} \mathrm{O}_{3}\right)$, dysprosium oxide $\left(\mathrm{Dy}_{2} \mathrm{O}_{3}\right)$, etc. In this paper, several common magneto-optical effects were briefly introduced, and the basic principles of Faraday effect and Kerr effect were illustrated in detail. In addition, the research progress, all-sides properties and application prospects of magneto-optical transparent ceramics were mainly reviewed. The properties of different kinds of magneto-optical transparent ceramics were compared and analyzed, and the existing problems as well as the research prospects were also proposed.
\end{abstract}

Key words: magneto-optical transparent ceramics; Faraday isolator; research progress; prospect; review

收稿日期: 2017-04-17; 收到修改稿日期：2017-05-11

基金项目: 国家自然科学基金(61575212) National Natural Science Foundation of China (61575212)

作者简介: 李 江(1977-), 男, 研究员. E-mail: lijiang@mail.sic.ac.cn 
磁光材料是一种从紫外到红外波段具有磁光效 应的光信息功能材料。利用这类材料的磁光特性以 及磁、光、电的相互作用和转换, 可以制成具有各 种功能的光学器件, 在激光、光纤通信、光纤传感 等高科技领域得到广泛应用。自发现磁光效应以来, 随着人们对磁光效应的研究和应用不断地深入和拓 展, 各类磁光材料相继涌现并得到快速的发展。与 此同时, 随着磁光器件不断朝着高功率、小型化、 低成本等方向发展, 对磁光材料的性能要求也越来 越高, 主要包括高 Verdet 常数、大尺寸、高光学质 量、高热导率和高激光损伤阈值等 ${ }^{[1]}$ 。

常见的磁光材料主要包括磁光玻璃、磁光晶体、 磁光陶瓷等。其中磁光玻璃和磁光晶体是两种传统 的磁光材料, 在磁光调制器 ${ }^{[2-4]}$ 、光纤电流传感器 ${ }^{[5]}$ 、 磁光隔离器 ${ }^{[6]}$ 等磁光器件中已获得较多应用。磁光 玻璃分为顺磁玻璃和逆磁玻璃两种 ${ }^{[7-14]}$, 其中顺磁 玻璃的 Verdet 常数较大, 可以提高磁光效应的灵敏 度，因而得到广泛使用。然而，磁光玻璃的热导率较 低, 热稳定性较差, 使用时容易形成热损伤, 无法 承受较高的激光功率, 因此应用范围受到较大的限 制。和磁光玻璃相比, 磁光晶体材料具有更高的热 导率，有效地避免了使用过程中的热效应问题，同 时其磁光性能也优于磁光玻璃, 在制备小尺寸的磁 光器件方面更具有优势。磁光晶体材料主要包括钇

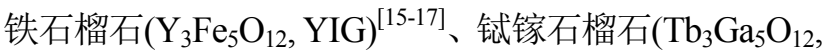
$\mathrm{TGG})^{[18-21]}$ 、铽铝石榴石 $\left(\mathrm{Tb}_{3} \mathrm{Al}_{5} \mathrm{O}_{12}, \mathrm{TAG}\right)^{[22-25]}$ 等, 但 是晶体材料也有其固有的缺陷，如不宜制成大体积 块材，制备周期较长，成本也相对较高等，这些问 题都限制了磁光晶体的应用范围。

近年来，随着透明陶瓷制备技术的快速发展， 透明陶瓷在力学、光学、热学等方面的优势日益凸 显 ${ }^{[26-31]}$, 也为磁光材料的发展提供了新的途径。磁 光陶瓷正成为近年来出现的一种新型的磁光材料。 磁光陶瓷的热导率和磁光晶体相当, 热扩散性能较 好, 可以有效防止激光过程中的热损伤。和晶体相 比, 磁光陶瓷材料更容易获得较大的尺寸, 能够做 成大口径的磁光元件; 并且其断裂㓞性高, 抗热震 性能好。这些性能上的优势满足了高功率激光器对 磁光材料的性能要求, 使得磁光陶瓷具有很好的应 用前景。

\section{1 磁光效应}

磁光效应是指在外加磁场的作用下，物质的电 磁特性(如磁导率、磁化强度、磁畴结构等)发生变 化, 使得光波在其内部的传输特性(如偏振状态、相
位、光强、传输方向等)也发生变化的现象 ${ }^{[32]}$ 。磁光 效应的种类较多，主要包括法拉第效应、克尔效应、 塞曼效应、科顿-穆顿效应等，其中法拉第效应和克 尔效应是目前研究和应用最为广泛的磁光效应，下 面将对这两种磁光效应做简单介绍。

\section{1 法拉第效应}

法拉第效应是指一束线偏振光通过置于磁场中 的介质时, 透射光的偏振方向发生旋转的现象, 如 图 1 所示。偏转角度为 $\theta$, 磁场强度为 $B$, 样品长度 为 $d$, 它们之间的关系如下:

$$
\theta=V B d
$$

其中, $V$ 是维尔德常数 ${ }^{[33]}$, 是物质固有的比例系数, 单位是 $\mathrm{rad} /(\mathrm{T} \cdot \mathrm{m})$ 。维尔德常数有正负之分, 当光的 传播方向与磁场方向一致时, $V$ 取正值，这时顺着光 的传播方向看去, $\theta$ 角度是左旋的; 当光的传播方向 与磁场方向相反时, $V$ 取负值, 这时顺着光的传播方 向看去, $\theta$ 角度是右旋的 ${ }^{[34]}$ 。法拉第效应具有非互易 性, 即磁致旋光的旋转方向和光的传播方向无关, 而由磁场的方向决定，当光线正反两次通过磁光材 料时, 透射光的旋转角将加倍, 这一点和自然旋光 不同 ${ }^{[35]}$ 。法拉第效应的应用非常广泛, 利用法拉第 效应原理可以制作光隔离器、回转器、磁光开关等 磁光器件。

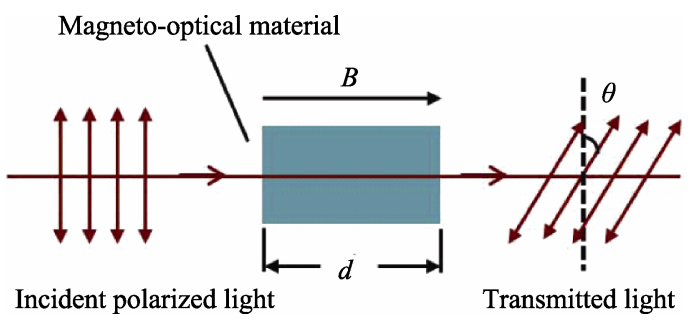

图 1 法拉第效应示意图

Fig. 1 Diagram of Faraday effect

\section{2 克尔效应}

克尔效应是指偏振光在磁光介质表面反射时, 反射光偏振面相对于入射光偏振面转过一定角度的 现象。根据外加磁场方向的不同，克尔效应分为极 向、纵向和横向三种，如图 2 所示。极向克尔效应， 即磁化强度与介质表面垂直时的克尔效应; 横向克 尔效应，即磁化强度与介质表面平行，但垂直于光 的入射面的克尔效应; 纵向克尔效应，即磁化强度 既平行于介质表面又平行于光入射面时的克尔效 应。克尔效应的一个重要应用是观察铁磁体的磁畴, 由于不同磁畴的自发磁化方向不同，使得入射偏振 光产生大小、方向不同的偏振面旋转, 再经过检偏 器后就出现了与磁畴对应的明暗不同的区域。 


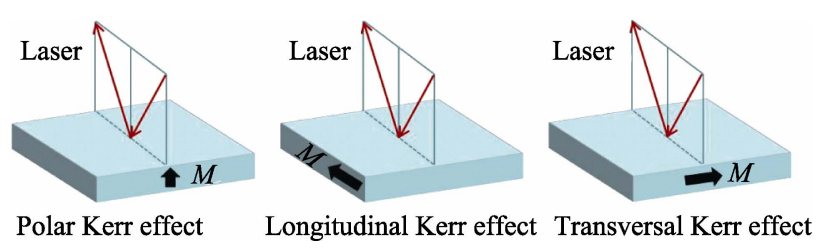

图 2 克尔效应示意图

Fig. 2 Diagram of Kerr effect

\section{2 磁光陶瓷}

一般的陶瓷材料是不透明的，这是因为在陶瓷 材料中存在大量晶界、气孔和杂质, 当入射光照射 时容易产生散射和吸收, 使光几乎无法通过。随着 粉体制备技术和陶瓷烧结技术的发展，透明陶瓷的 制备已经成为现实, 并且随着技术的不断改进，陶 瓷的光学质量得到很大提高, 几乎可与晶体媲美。 目前已经出现的磁光陶瓷材料主要有铽镓石榴石 (TGG) 陶瓷、铽铝石榴石 (TAG) 陶瓷等，近年来还 出现了关于倍半氧化物磁光陶瓷, 如氧化铽 $\left(\mathrm{Tb}_{2} \mathrm{O}_{3}\right)$ 陶瓷、氧化钬 $\left(\mathrm{Ho}_{2} \mathrm{O}_{3}\right)$ 陶瓷、氧化镝 $\left(\mathrm{Dy}_{2} \mathrm{O}_{3}\right)$ 陶瓷等的 研究报道, 下面对这几种磁光陶瓷材料做逐一介绍。

\section{1 铽镓石榴石(TGG)陶瓷}

琙镓石榴石(TGG) 是一种应用于可见光及近红 外波段的顺磁性法拉第磁光材料, 属石榴石立方相 晶体, 由氧原子堆积出晶体结构, $\mathrm{Tb}^{3+}$ 占据氧十二 面体空隙 $\left(\mathrm{C}\right.$ 位), 两个 $\mathrm{Ga}^{3+}$ 占据八面体空隙( $\mathrm{A}$ 位)、 三个 $\mathrm{Ga}^{3+}$ 占据四面体空隙(D 位)。TGG 单晶在可见 光和近红外波段具有较高的 Verdet 常数和较低的吸 收系数, 并且激光损伤阈值较高, 是目前 400 1100 nm (不包括 470 500 nm) 波段应用最多的磁光材料之一, 被广泛地应用于 YAG 激光、掺钛蓝宝石可调谐激 光、环形激光和种子注入的激光器。但是 TGG 晶体 在生长过程中常伴有 $\mathrm{Ga}_{2} \mathrm{O}_{3}$ 的挥发, 使其组分偏离 化学计量比, 从而出现较多缺陷及开裂等问题 ${ }^{[36]}$ 。 而陶瓷的制备过程无需熔化原料, 这样可以有效减 少 $\mathrm{Ga}_{2} \mathrm{O}_{3}$ 的挥发, 避免组分偏离。另外, 与 TGG 单 晶相比, TGG 陶瓷还具有成本低、断裂㓞性高、抗 热震性好、大尺寸等方面的优势 ${ }^{[1]}$, 能满足高功率激 光器对大口径磁光元件的需求。因此, $\mathrm{TGG}$ 陶瓷是 应用于高能以及高平均功率激光器的最理想的材料 之一。

日本科学家 Ikesue 制备了第一个 TGG 陶瓷样 品, 但是其光学质量并不高。2003 年, 俄罗斯科学 家 Khazanov 首次对 TGG 陶瓷进行了报道, 并阐述 了其应用在高功率激光器中的可能性 ${ }^{[37]}$ 。2004 年, Kagan 等 ${ }^{[38]}$ 对 TGG 陶瓷的热致双折射效应进行了 理论分析。但由于 TGG 陶瓷的光学质量不高, 其后
几年间没有关于 TGG 陶瓷的相关报道。随着透明陶 瓷制备技术的发展，TGG 陶瓷的光学质量也逐渐得 到提高。2007 年, Yasuhara 等 ${ }^{[33]}$ 首次对 TGG 陶瓷的 法拉第效应进行了报道, 他们测试了日本神岛化学 公司提供的 TGG 陶瓷样品在 $1053 \mathrm{~nm}$ 处的 Verdet 常 数随温度的变化, 并与 $\mathrm{TGG}$ 单晶做了比较, 如图 3 所示。结果表明, TGG 陶瓷的 Verdet 常数和 TGG 单 晶相近, 室温下的 Verdet 常数为 $36.4 \mathrm{rad} /(\mathrm{T} \cdot \mathrm{m})$, 液 氮温度下 $(77 \mathrm{~K})$ 的 Verdet 常数是室温时的 87 倍。

2011 年, Yoshida 等 $^{[1]}$ 对 TGG 陶瓷的光学性能、 散射损耗、激光损伤阈值以及法拉第效应进行了系 统研究。他们首先观察了 $\mathrm{TGG}$ 陶瓷的微观形貌, 发 现其晶粒粒径分布较为均匀, 粒径大小在 $0.3 \sim 3 \mu \mathrm{m}$ 之间。同时, 他们测试了 $\mathrm{TGG}$ 陶瓷样品在可见光及 近红外波段的光学透过率, 并和 TGG 单晶做了对 比, 如图 4 所示。从图 4 可以看到, 在 $>600 \mathrm{~nm}$ 波段, TGG 陶瓷和单晶的透过率几乎相等，均高于 $80 \%$;

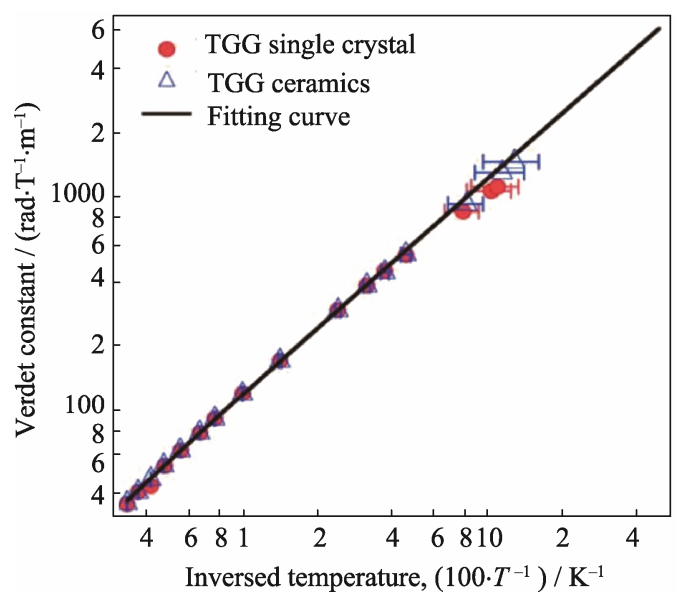

图 $3 \mathrm{TGG}$ 单晶和 $\mathrm{TGG}$ 陶瓷的 Verdet 常数随温度的变化 ${ }^{[33]}$ Fig. 3 Temperature dependence of Verdet constant of TGG single crystal and TGG ceramics ${ }^{[33]}$

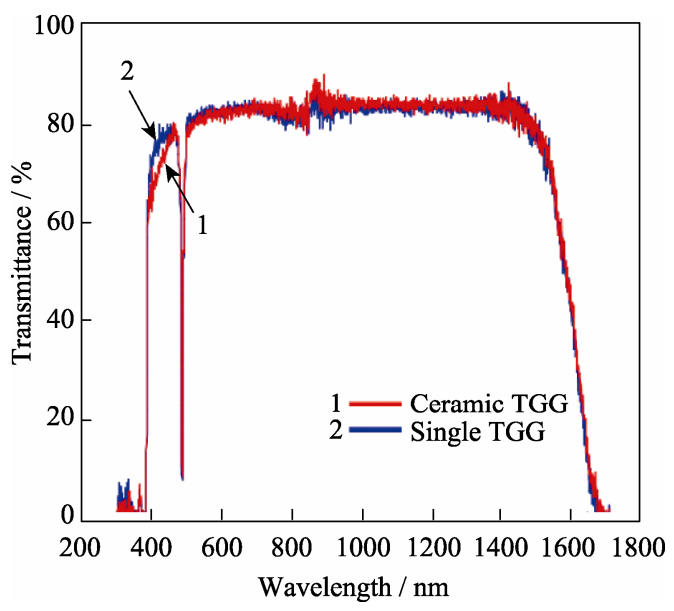

图 4 TGG 单晶和陶瓷的透过率曲线 ${ }^{[1]}$

Fig. 4 Transmission spectra of single crystal TGG and ceramic TGG samples ${ }^{[1]}$ 
在 $<600 \mathrm{~nm}$ 波段, $\mathrm{TGG}$ 陶瓷的透过率略低于单晶。 $480 \mathrm{~nm}$ 处的吸收峰对应于 $\mathrm{Tb}^{3+}$ 的 ${ }^{7} \mathrm{~F}_{6} \rightarrow{ }^{5} \mathrm{D}_{4}$ 跃迁。图 4 结果表明, TGG 陶瓷的光学质量已经可以和单晶相 媲美。利用积分球测试了 $\mathrm{TGG}$ 陶瓷和单晶在 632 和 $1064 \mathrm{~nm}$ 处的散射损耗, 如表 1 所示。结果表明, $632 \mathrm{~nm}$ 处 TGG 陶瓷的散射损耗是单晶的 2.4 倍, 有 待通过改进陶瓷制备工艺来降低; $1064 \mathrm{~nm}$ 处两者 的散射损耗相近。实验测得室温下 TGG 陶瓷在 $1064 \mathrm{~nm}$ 处的 Verdet 常数和 TGG 单晶相近, 消光比 超过 $35 \mathrm{~dB}$, 满足商用法拉第隔离器的使用要求。激 光损伤实验表明, TGG 陶瓷在 $\mathrm{Nd}$ : YAG 激光照射下 的激光损伤阈值和 TGG 单晶接近, 仍存在很大的 提升空间。

随着连续激光器和百浦激光器的平均功率不断 增大, 激光系统中光学元件的热效应变得越来越严 重。热致双折射效应会降低法拉第隔离器的消光比, 从而限制其在高功率激光器中的使用。因此, 磁光 材料在高功率激光辐照下的相关热学性能, 如热致 退偏效应、热透镜效应等也愈发受到关注, 以此可 以初步判断其在高功率激光器中的应用前景。基于 此, 2013 年, Yasuhara 等 ${ }^{[39]}$ 对 TGG 陶瓷材料在激光 辐照下的热致退偏效应、热透镜效应及热流稳定性 进行了系统研究。他们首先测试了 $\mathrm{TGG}$ 陶瓷在激光 辐照下的热致退偏比, 并和 TGG 单晶做了对比。如 图 5 所示, 外加磁场时 ( $45^{\circ}$ 法拉第旋转角), TGG 陶 瓷在 $257 \mathrm{~W}$ 激光功率下的退偏比为 $5.48 \times 10^{-4}$, 对应 的消光比为 $33 \mathrm{~dB}$, TGG 陶瓷和单晶的热致退偏几 乎相一致。此外, 他们测试了基于该陶瓷样品的法 拉第隔离器的热透镜焦距随激光功率的变化, 结果 表明 $257 \mathrm{~W}$ 激光功率下的热透镜焦距为 $9.5 \mathrm{~m}$, 由此 预计当激光功率为 $600 \mathrm{~W}$ 时, 热透镜焦距为 $4 \mathrm{~m}$ 。这 种热透镜效应完全可以通过球形透镜来校正。他们 还测试了在 $257 \mathrm{~W}$ 激光功率下, 法拉第偏转角以及 热致退偏的时间稳定性, 如图 6 所示。可以看到, 法 拉第偏转角在 $2 \mathrm{~min}$ 后就达到稳定状态, 退偏比在 整个实验过程中都能保持稳定, 这说明通过水冷装 置可以有效去除由激光辐照产生的热量, 从而保持 温度稳定性。

表 1 TGG 陶瓷和 TGG 单晶样品的散射损耗 ${ }^{[1]}$

Table 1 Optical scattering of ceramic and single crystal TGG samples $^{[1]}$

\begin{tabular}{ccccc}
\hline $\begin{array}{c}\text { Laser } \\
\text { wavelength } \\
\text { /nm }\end{array}$ & $\begin{array}{c}\text { F.S.surface } \\
\text { reflectivity }\end{array}$ & $\begin{array}{c}\text { Crystal } \\
\text { TGG } \\
18 \mathrm{~mm}\end{array}$ & $\begin{array}{c}\text { Ceramic } \\
\text { TGG } \\
20 \mathrm{~mm}\end{array}$ & $\begin{array}{c}\text { Scattering } \\
\text { ratio } \\
\text { (Ceramic/Crystal) }\end{array}$ \\
\hline 632 & $3.45 \times 10^{-2}$ & $\begin{array}{c}1.4 \times 10^{-3} \\
\left(1 \times 10^{-3} \mathrm{~cm}^{-1}\right)\end{array}$ & $\begin{array}{c}4.8 \times 10^{-3} \\
\left(2.4 \times 10^{-3} \mathrm{~cm}^{-1}\right)\end{array}$ & 2.4 \\
1064 & $3.36 \times 10^{-2}$ & $\begin{array}{c}1 \times 10^{-3} \\
\left(5 \times 10^{-4} \mathrm{~cm}^{-1}\right)\end{array}$ & $\begin{array}{c}1 \times 10^{-3} \\
\left(5 \times 10^{-4} \mathrm{~cm}^{-1}\right)\end{array}$ & 1.0 \\
\hline
\end{tabular}

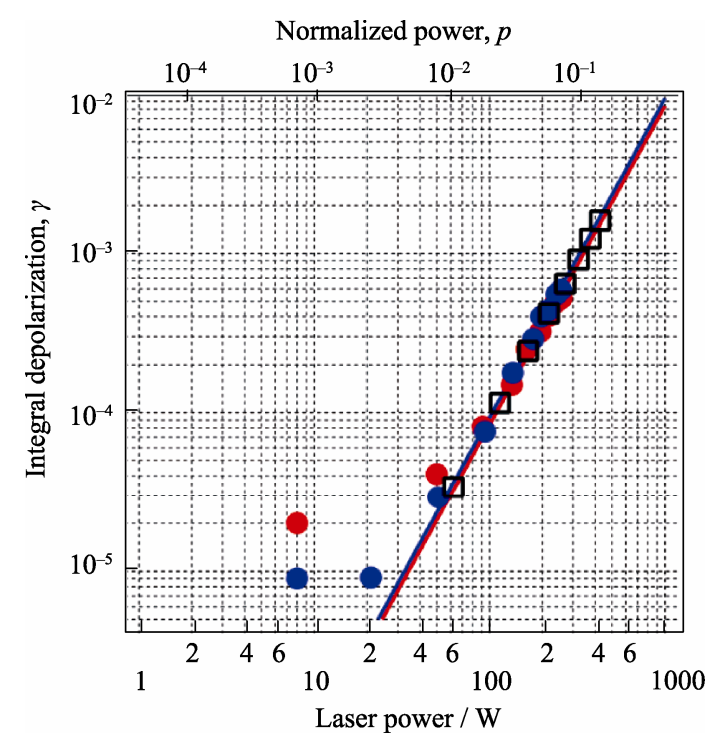

图 5 退偏比随激光功率的变化 ${ }^{[39]}$

Fig. 5 Experimental results of depolarization as a function of laser power

Red and blue circles denote results for the TGG ceramic with and without a magnet field. Open squares denote the calculated result for the $\mathrm{TGG}^{[39]}$

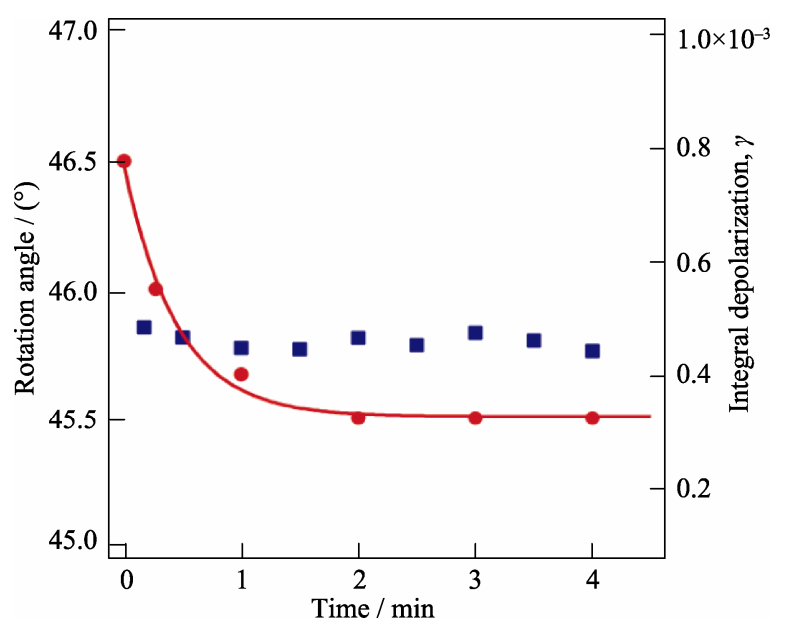

图 6 退偏比(蓝色方块)和法拉第旋转角(红色实心圆)随时 间的变化 ${ }^{[39]}$

Fig. 6 Time dependence of depolarization (blue squares) and Faraday rotation angle (red circles) ${ }^{[39]}$

以上结果均表明，基于 $\mathrm{TGG}$ 陶瓷的法拉第隔离 器已基本满足在高功率激光下服役的要求。随着激 光功率的不断增大以及对光束质量要求的不断提高, 人们采用不同方法来降低热致退偏效应的影响, 采 用最多的是通过补偿装置来减小热致退偏, 从而提 高法拉第隔离器的消光比。Snetkov 等 ${ }^{[40]}$ 首次在基 于 TGG 陶瓷的法拉第隔离器中, 利用另外一个 TGG 陶瓷样品和石英旋转器组成的补偿元件对其进行热 致退偏补偿, 使其在 $300 \mathrm{~W}$ 激光功率下的消光比从 $30 \mathrm{~dB}$ 增大到 $38 \mathrm{~dB}$ 。通过理论计算, 采用这样的补 偿装置可使其在 $2 \mathrm{~kW}$ 激光功率下的消光比达到 $30 \mathrm{~dB}$, 从而进一步表明 $\mathrm{TGG}$ 陶瓷在高功率激光器 
中的良好的应用前景。

\section{2 铽铝石榴石(TAG)陶瓷}

琙铝石榴石(TAG)也是一种应用于可见及近红 外波段的法拉第磁光材料, 与 $\mathrm{TGG}$ 具有相同的石 榴石结构, 相近的光学性能和热学性能, 但是磁光 性能更为优异，其 Verdet 常数比 TGG 高 30\% 50\% 左右。因此, TAG 是比 TGG 更具有发展前景的磁光 材料。TAG 的分子式为 $\mathrm{Tb}_{3} \mathrm{Al}_{5} \mathrm{O}_{12}$, 具有石榴石立方 相晶体结构。氧原子堆积出晶体结构, $\mathrm{Tb}^{3+}$ 占据氧十 二面体空隙 $\left(\mathrm{C}\right.$ 位), 两个 $\mathrm{Al}^{3+}$ 占据八面体空隙 $(\mathrm{A}$ 位)、三个 $\mathrm{Al}^{3+}$ 占据四面体空隙(D 位)。由于 $\mathrm{TAG}$ 的 非一致熔融特性, 晶体制备非常困难, 所以一直未 获得实际应用。而陶瓷的制备温度低于 TAG 的熔点 温度, 有效避免了 $\mathrm{TAG}$ 晶体生长过程中的非一致熔 融问题，使 TAG 介质的优良特性得以实现。

2011 年, 中国科学院上海光机所的 $\operatorname{Lin}$ 等 $^{[41]}$ 首 次通过固相反应结合真空烧结技术制备出了 $\mathrm{TAG}$ 磁光透明陶瓷。他们将陶瓷素坏在不同温度下保温 $5 \mathrm{~h}$ 进行真空烧结, 当烧结温度为 $1650^{\circ} \mathrm{C}$ 时, 得到的 陶瓷样品的光学透过率在 $500 \sim 1500 \mathrm{~nm}$ 波段接近 $70 \%$, 如图 7 所示。实验测得该 $\mathrm{TAG}$ 陶瓷样品在 室温下的热导率为 $6.5 \mathrm{~W} /(\mathrm{m} \cdot \mathrm{K})$, 在 $632.8 \mathrm{~nm}$ 处 的 Verdet 常数为 $-172.72 \mathrm{rad} /(\mathrm{T} \cdot \mathrm{m})$, 比 $\mathrm{TGG}$ 单晶 $(-134 \mathrm{rad} /(\mathrm{T} \cdot \mathrm{m}))$ 高 $28.9 \%$ 。以上结果表明, TAG 陶瓷 比商用 $\mathrm{TGG}$ 单晶的磁光性能更为优异, 但是仍需 提高其光学质量来满足应用需求。

Chen 等 ${ }^{[42]}$ 通过优化烧结助剂发现, TEOS 结合 $\mathrm{MgO}$ 作为烧结助剂能够提高 $\mathrm{TAG}$ 透明陶瓷的光学 质量。研究发现, 当 TEOS 添加量为 $0.4 \mathrm{wt} \%, \mathrm{MgO}$ 添加量为 $0.1 \mathrm{wt} \%$ 时, 得到的 $\mathrm{TAG}$ 陶瓷的光学透过 率在 500 1500 nm 波段超过了 $80 \%$, 光学质量得到

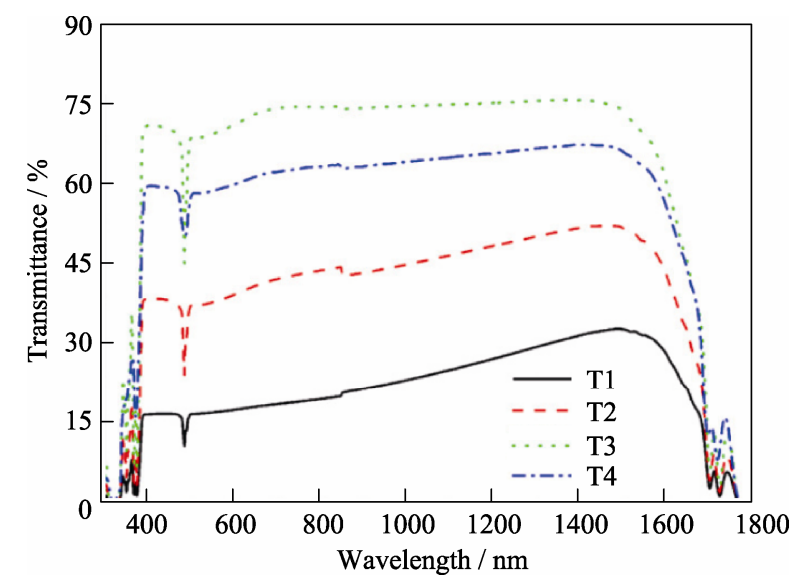

图 7 不同温度下烧结制备的 $\mathrm{TAG}$ 陶瓷的光学透过率 ${ }^{[41]}$

Fig. 7 Optical transmittance of the mirror polished transparent ceramics sintered at different temperatures ${ }^{[41]}$ $\mathrm{T} 1-1550^{\circ} \mathrm{C} ; \mathrm{T} 2-1600^{\circ} \mathrm{C} ; \mathrm{T} 3-1650^{\circ} \mathrm{C} ; \mathrm{T} 4-1700^{\circ} \mathrm{C}$
了较大的提高。此外, 他们还发现 $\mathrm{MgO}$ 的添加量对 TAG 陶瓷的磁光性能并无影响。Dai 等 ${ }^{[43]}$ 发现当烧 结温度达到 $1725^{\circ} \mathrm{C}$ 时, TAG 陶瓷样品会发生玻璃化, 变为无定型相, 但仍然保持较好的光学性能和磁光 性能, 其光学透过率在 600 1500 nm 波段超过 $80 \%$, 在 $633 \mathrm{~nm}$ 处的 Verdet 常数为 $-179.3 \mathrm{rad} /(\mathrm{T} \cdot \mathrm{m})$ 。

除了固相反应法制备 TAG 磁光透明陶瓷之外, 戴佳卫等 ${ }^{[4]}$ 提出了一种新型的制备 $\mathrm{TAG}$ 纳米粉体 及磁光透明陶瓷的方法, 即先采用共沉淀法合成 TAG 纳米粉体，再采用真空预烧结和热等静压烧结 相结合来制备 TAG 磁光透明陶瓷。与固相法相比, 该方法合成的粉体分散性能较好，颗粒尺寸细小， 比表面积大, 具有较好的烧结活性, 有利于透明陶 瓷的后期烧结。戴佳卫等 ${ }^{[45]}$ 还提出了一种非水基凝 胶注模成型制备琙铝石榴石基磁光透明陶瓷的方法, 该方法采用非水溶剂来制备陶瓷浆料，固相含量高 达 $60 \mathrm{vol} \%$, 浆料性能稳定并且具有较好的流动性, 非常适合凝胶注模成型，且制备工艺简单，成本低 廉，具有较强的实用性。通过该方法得到的陶瓷素 坏形状可控, 成分分布均匀, 具有较高的致密度, 可制备形状复杂的磁光陶瓷零件。

$\mathrm{TAG}$ 的磁光效应主要来源于其所含的 $\mathrm{Tb}^{3+}$, 因 此可以通过不同稀土离子取代 $\mathrm{Tb}^{3+}$ 来改变其磁光特 性。Chen 等 ${ }^{[46]}$ 用抗磁性离子 $\mathrm{Y}^{3+}$ 来部分取代 $\mathrm{Tb}^{3+}$, 采用固相反应法制备得到化学式为 $\left(\mathrm{Tb}_{0.8} \mathrm{Y}_{0.2}\right)_{3} \mathrm{Al}_{5} \mathrm{O}_{12}$ 的磁光透明陶瓷，测得其在 $632.8 \mathrm{~nm}$ 处的 Verdet 常 数仅为 $-108.79 \mathrm{rad} /(\mathrm{T} \cdot \mathrm{m})$, 比 TAG 陶瓷低 $37 \%$, 这 说明抗磁性离子掺杂会对 $\mathrm{TAG}$ 的磁光性能产生不 利影响。此外, 他们还用顺磁性离子 $\mathrm{Ce}^{3+}$ 作为掺杂 离子, 制备了化学式为 $\left(\mathrm{Tb}_{0.997} \mathrm{Ce}_{0.003}\right)_{3} \mathrm{Al}_{5} \mathrm{O}_{12}$ 的磁光 透明陶瓷 ${ }^{[47]}$ 。该磁光陶瓷在可见和近红外波段的光 学透过率低于 $70 \%$, 并且在 400 500 nm 波段出现了 由 $\mathrm{Ce}^{3+}: 4 \mathrm{f} \rightarrow 5 \mathrm{~d}_{1}$ 跃迁引起的较宽的吸收峰。磁光性 能测试结果表明, Ce:TAG 透明陶瓷在 $632.8 \mathrm{~nm}$ 波长 处的 Verdet 常数为 $-199.55 /(\mathrm{T} \cdot \mathrm{m})$, 比 TAG 陶瓷的 Verdet 常数还要大 $16 \%$ 左右, 说明掺入顺磁性 $\mathrm{Ce}^{3+}$ 离子对 TAG 样品的磁光性能有较大的提高。除了 $\mathrm{Tb}$ 位取代之外，也可以通过 $\mathrm{Al}$ 位取代对 $\mathrm{TAG}$ 陶瓷 的磁光性能进行调控。Furuse 等 ${ }^{[48]}$ 用 $\mathrm{Ti}$ 离子取代 $\mathrm{Al}$ 离子, 制备了化学式为 $\mathrm{Tb}_{3}\left(\mathrm{Al}_{0.992} \mathrm{Ti}_{0.008}\right)_{5} \mathrm{O}_{12}$ 的磁 光透明陶瓷, 测得其在 $632.8 \mathrm{~nm}$ 和 $1064 \mathrm{~nm}$ 处的 Verdet 常数分别为 184 和 $53 \mathrm{rad} /(\mathrm{T} \cdot \mathrm{m})$, 高于 $\mathrm{TGG}$ 和 $\mathrm{TAG}$ 陶瓷。他们认为磁光性能的提高与 $\mathrm{Ti}$ 离子 的引入改变了 $\mathrm{TAG}$ 的晶体场有关。

Starobor 等 ${ }^{[49]}$ 对 TGG、TAG、Ce:TAG(0.1at $\left.\%\right)$ 
透明陶瓷在高功率隔离器中的应用作了比较, 热致 退偏实验结果如图 8 所示。从图 8 可以看到, 在较 低功率值时, Ce:TAG 陶瓷样品的热致退偏值较大, 隔离效果较差; 而当功率升到 $100 \mathrm{~W}$ 以上时, Ce:TAG 陶瓷样品的退偏值增加的幅度较小, 此时 它与 TAG 陶瓷样品同时优于 TGG 样品。热透镜效 应对比实验结果如图 9 所示, 所用激光束的半径为 $24 \mathrm{~mm}$, 从图中可以看出 $\mathrm{TAG}$ 与 $\mathrm{TGG}$ 透明陶瓷样 品的热透镜效应相当, 且比 Ce:TAG 要高两倍。

根据文献报道, 将 TGG、TAG、Ce:TAG 陶瓷 样品和 $\mathrm{TGG}$ 单晶在高功率法拉第隔离器中应用时 的消光比和热透镜的实验结果 ${ }^{[48]}$ 列于表 2 , 可以看 到, TGG 陶瓷与 TGG 晶体相比隔离度还有一定差 距, 其他几乎没有差距。TAG 和 Ce:TAG 透明陶瓷 的隔离度测量功率较低, 但是通过计算可以估计当 功率达到千瓦级时, TAG 陶瓷样品的消光比可以保 持在 $30 \mathrm{~dB}$ 以上。综合比较可以看出, 目前为止 $\mathrm{TAG}$ 透明陶瓷与 $\mathrm{TGG}$ 晶体的隔离度相当, 通过退火处 理可以将其隔离度提高几倍, 因此 TAG 透明陶瓷可 用于制备千瓦级的法拉第隔离器 ${ }^{[9-54]}$ 。

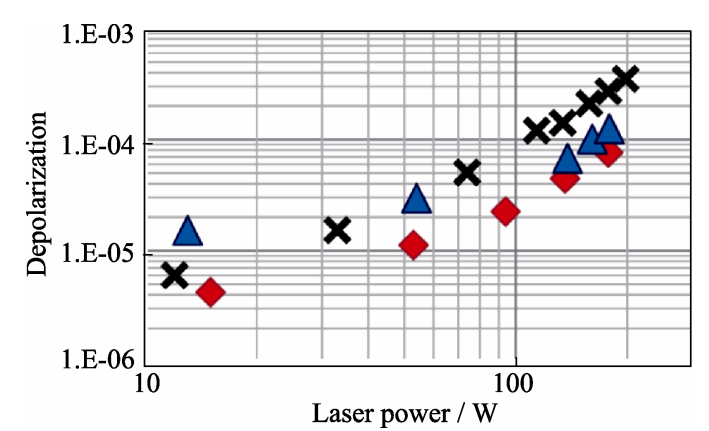

图 $8 \mathrm{TGG}(\boldsymbol{x}) 、 \mathrm{TAG}(\bullet)$ 及 $\mathrm{Ce}: \mathrm{TAG}(0.1 \mathrm{at} \%)(\boldsymbol{\Delta})$ 透明陶瓷样 品的热致退偏随辐射功率的变化图 ${ }^{[49]}$

Fig. 8 Power dependence of depolarization for TGG( $\times$ ), $\operatorname{TAG}(\bullet)$, $\mathrm{Ce}: \operatorname{TAG}(\mathbf{\Delta})^{[49]}$

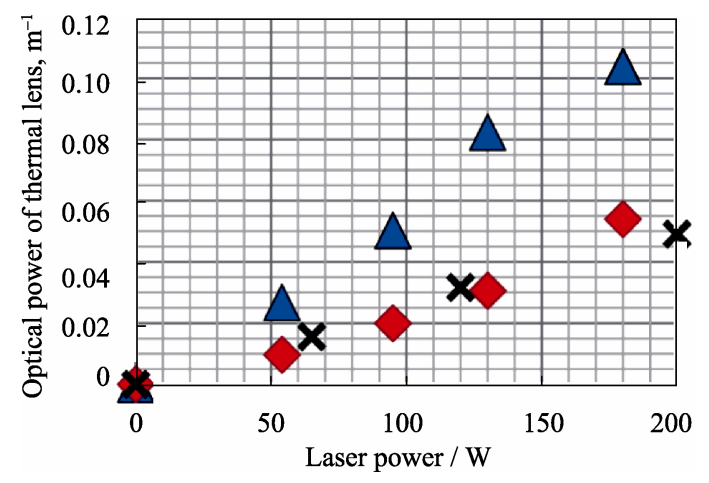

图 $9 \mathrm{TGG}(\boldsymbol{\times}) 、 \mathrm{TAG}(\bullet)$ 及 $\mathrm{Ce}: \mathrm{TAG}(0.1 \mathrm{at} \%)(\boldsymbol{\Delta})$ 透明陶瓷样 品的热透镜光功率随辐射功率的变化图 ${ }^{[49]}$

Fig. 9 Optical power of thermal lens versus rudiation power for $\operatorname{TGG}(\times), \operatorname{TAG}(\bullet), \operatorname{Ce}: \operatorname{TAG}(\boldsymbol{\Delta})^{[49]}$
表 2 基于不同磁光介质的法拉第隔离器的参数比较 ${ }^{[48]}$

Table 2 Ceramic-based FI characteristics ${ }^{[48]}$

\begin{tabular}{cccc}
\hline \multirow{2}{*}{ Medium } & $\begin{array}{c}\text { Isolation ratio } \\
\text { @ laser power }\end{array}$ & $\begin{array}{c}\text { Thermal lens } \\
\text { @ laser power }\end{array}$ & $\begin{array}{c}\text { Water } \\
\text { cooling }\end{array}$ \\
\hline TGG crystal & $30 \mathrm{~dB} @ 650 \mathrm{~W}$ & $6.5 \mathrm{~m} @ 340 \mathrm{~W}$ & Optional \\
TGG ceramics & $30 \mathrm{~dB} @ 340 \mathrm{~W}$ & $6.5 \mathrm{~m} @ 340 \mathrm{~W}$ & Optional \\
TAG ceramics & $38 \mathrm{~dB} @ 300 \mathrm{~W}$ & $8 \mathrm{~m} @ 300 \mathrm{~W}$ & Necessary \\
Ce:TAG ceramics $(0.1 \mathrm{at} \%)$ & $31 \mathrm{~dB} @ 300 \mathrm{~W}$ & $3.8 \mathrm{~m} @ 300 \mathrm{~W}$ & Necessary \\
\hline
\end{tabular}

\section{3 倍半氧化物磁光陶瓷}

倍半氧化物通常具有较高的热导率, 是一种很 好的应用于高能激光器的激光增益介质。近年来, 随着对磁光材料研究的深入, 人们在许多倍半氧化 物材料中发现了磁光效应。本文将对铽掺杂氧化钎 $\left(\mathrm{Tb}^{3+}: \mathrm{Y}_{2} \mathrm{O}_{3}\right)$ 、氧化钬 $\left(\mathrm{Ho}_{2} \mathrm{O}_{3}\right)$ 、氧化镝 $\left(\mathrm{Dy}_{2} \mathrm{O}_{3}\right)$ 等几种 常见的倍半氧化物磁光陶瓷作介绍。

氧化钎 $\left(\mathrm{Y}_{2} \mathrm{O}_{3}\right)$ 是一种很好的基质材料, 这是 因为它易被不同的稀土金属离子掺杂，同时制备技 术相对成熟, 目前已达到激光级水平。Snetkov 等 ${ }^{[5]}$ 通过自蔓延高温合成法制备了不同掺杂浓度的 $\mathrm{Tb}^{3+}: \mathrm{Y}_{2} \mathrm{O}_{3}$ 粉体，再通过热压烧结制备得到了 $\mathrm{Tb}^{3+}: \mathrm{Y}_{2} \mathrm{O}_{3}$ 陶瓷, 其实物照片及透过率曲线如图 10 所示。从图 10 可以看到, 掺杂浓度 $10 \%$ 的 $\mathrm{Tb}^{3+}: \mathrm{Y}_{2} \mathrm{O}_{3}$ 陶瓷的光学质量最好, 它在 $1064 \mathrm{~nm}$ 波长处的光学 透过率达到 $79.3 \%$ 。实现完全掺杂的 $\mathrm{Tb}_{2} \mathrm{O}_{3}$ 陶瓷样 品在 $1064 \mathrm{~nm}$ 波长处的光学透过率达到 $63.4 \%$, 低 于其理论透过率 $81.1 \%$, 有待通过进一步优化制备 工艺来提高。此外, 他们对 $\mathrm{Tb}^{3+}: \mathrm{Y}_{2} \mathrm{O}_{3}$ 陶瓷的磁光性 能进行了研究, 测试了它们在 405、447、530、633、 809、980、1075、1310 和 $1561 \mathrm{~nm}$ 波长处的 Verdet 常数, 并与 TGG 作了对比, 如图 11 所示。从图 11 可以看到, 随着 $\mathrm{Tb}^{3+}$ 掺杂浓度的增加, $\mathrm{Tb}^{3+}: \mathrm{Y}_{2} \mathrm{O}_{3}$ 陶 瓷的 Verdet 常数也逐渐增大。当掺杂浓度为 $30 \%$ 时, 其 Verdet 常数和 $\mathrm{TGG}$ 相当。 $\mathrm{Tb}_{2} \mathrm{O}_{3}$ 陶瓷在 380 1750 nm 波段的 Verdet 常数是 TGG 的 3 倍以上, 这是迄今已 知的 Verdet 常数最高的透明材料。Furuse 等 ${ }^{[56]}$ 通过 SPS 烧结方法成功制备了 $\mathrm{Ho}_{2} \mathrm{O}_{3}$ 磁光透明陶瓷, 测得其 在 $1064 \mathrm{~nm}$ 波长处的 Verdet 常数为 $-46.3 \mathrm{rad} /(\mathrm{T} \cdot \mathrm{m})$, 和 $\mathrm{TAG}$ 接近, 是 $\mathrm{TGG}$ 的 1.3 倍左右。它在 $1 \mu \mathrm{m}$ 处的 透过率为 $60 \%$ 左右, 光学质量有待进一步提高。 Morales 等 ${ }^{[57]}$ 通过 SPS 烧结方法成功制备了 $\mathrm{Dy}_{2} \mathrm{O}_{3}$ 磁光透明陶瓷，在 $633 \mathrm{~nm}$ 处的透过率为 $50 \%$ 左右， Verdet 常数为 $-300 \mathrm{rad} /(\mathrm{T} \cdot \mathrm{m})$, 是 TGG 的 2.25 倍。但 是由于 $\mathrm{Dy}^{3+}$ 在 $1 \mu \mathrm{m}$ 附近存在较强的吸收峰, 它难以 应用于基于掺杂 $\mathrm{Yb}$ 和 $\mathrm{Nd}$ 的基质的激光器中。 


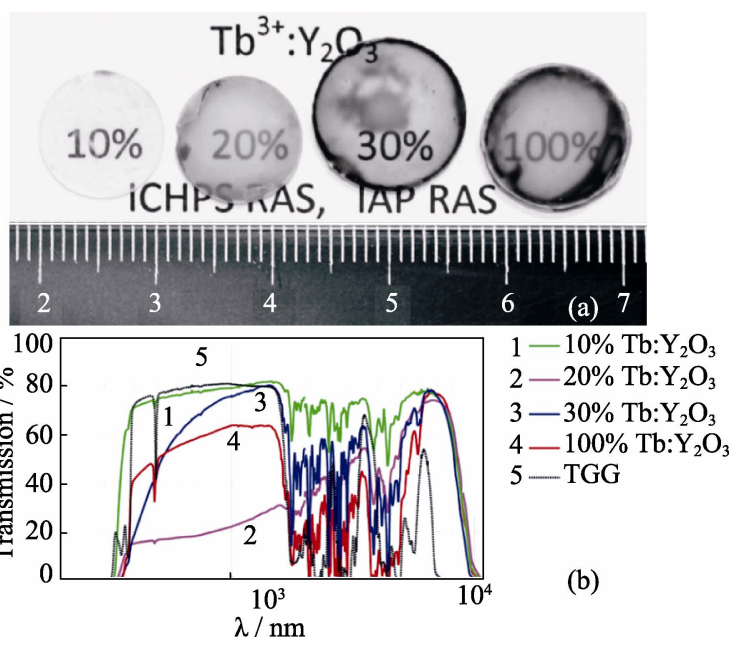

图 $10 \mathrm{~Tb}^{3+}: \mathrm{Y}_{2} \mathrm{O}_{3}$ 陶瓷的(a)实物照片和(b)透过率曲线 ${ }^{[55]}$

Fig. 10 (a) Photograph and (b) transmission spectra of $\mathrm{Tb}^{3+}: \mathrm{Y}_{2} \mathrm{O}_{3}$ ceramics $^{[55]}$
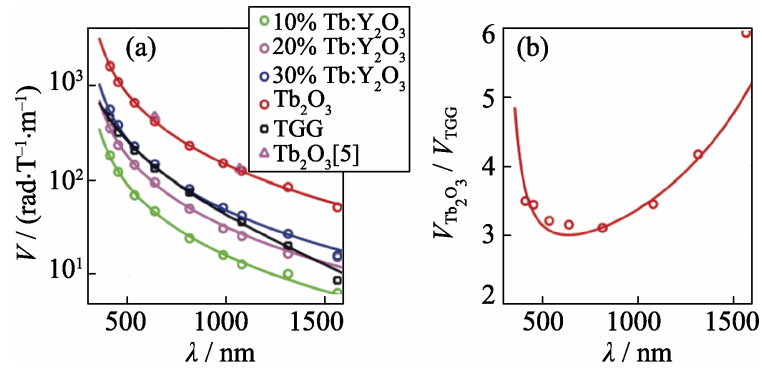

图 11 (a)不同掺杂浓度的 $\mathrm{Tb}^{3+}: \mathrm{Y}_{2} \mathrm{O}_{3}$ 陶瓷和 $\mathrm{TGG}$ 单晶在不同 波长处的 Verdet 常数, (b) $\mathrm{Tb}_{2} \mathrm{O}_{3}$ 和 TGG 的 Verdet 常数之比 ${ }^{[55]}$ 与波长的关系

Fig. 11 (a) Wavelength dependence of Verdet constant of $\mathrm{Tb}^{3+}: \mathrm{Y}_{2} \mathrm{O}_{3}$ for $10 \%, 20 \%$, and $30 \%$ concentrations of $\mathrm{Tb}^{3+}$ ions, $\mathrm{Tb}_{2} \mathrm{O}_{3}$ ceramics, and TGG single crystal; (b) $V_{\mathrm{Tb}_{2} \mathrm{O}_{3}} / V_{\mathrm{TGG}}$ ratio versus wavelength $^{[55]}$

\section{3 结论与展望}

随着连续激光器和泵浦激光器的平均功率的不 断增大, 对法拉第隔离器的性能要求也越来越高。 作为光隔离器的核心组成部分, 磁光材料的性能决 定了器件的性能, 因而它的发展也备受关注。磁光 陶瓷作为一种新型的磁光介质材料, 在力学、热学、 尺寸等方面的优势日益凸显, 这些性能上的优势满 足了高功率激光器对磁光材料的性能要求, 使得磁 光陶瓷具有很好的应用前景。

通过与商用 $\mathrm{TGG}$ 单晶的性能对比, 我们认为 TGG 和 TAG 陶瓷这两种材料已基本满足法拉第隔 离器的使用要求。未来研究的重点: 一是要通过制 备工艺的优化来进一步提高这两种材料的光学质量, 降低其吸收损耗, 以进一步满足高功率激光器的要 求; 二是通过不同种类稀土离子的掺杂对其进行磁 光性能、热学性能和机械性能等各项性能的调控, 以满足不同的商用需求。倍半氧化物磁光透明陶瓷
的研究目前还较少，其中 $\mathrm{Tb}_{2} \mathrm{O}_{3}$ 陶瓷表现出非常优 异的磁光性能，具有很好的应用前景，但是其材料 的可控制备及光学性能的改善还需要进一步研究。

\section{参考文献:}

[1] YOSHIDA H, TSUBAKIMOTO K, FUJIMOTO Y, et al. Optical properties and Faraday effect of ceramic terbium gallium garnet for a room temperature Faraday rotator. Opt. Express, 2011, 19(16): $15181-15187$

[2] ZU P, CHAN C C, LEW W S, et al. High extinction ratio magnetooptical fiber modulator based on nanoparticle magnetic fluids. IEEE Photonics J., 2012, 4(4): 1140-1146.

[3] DUFFY R M, NETTERFIELD R P. Design of Faraday rotators and modulators. Rev. Sci. Instrum., 1984, 55(5): 743-746.

[4] TATAM R P, BERWICK M, JONES J D C, et al. Faraday effect magnetometry utilizing high Verdet constant glass. Appl. Phys. Lett., 1987, 51(11): 864-866.

[5] LEE B. Review of the present status of optical fiber sensors. Opt. Fiber Technol., 2003, 9(2): 57-79.

[6] SHOJI Y, MIZUMOTO T, YOKOI H, et al. Magneto-optical isolator with silicon waveguides fabricated by direct bonding. Appl. Phys. Lett., 2008, 92(7): 071117.

[7] ZABLUDA V, POZELUYKO A, EDELMAN I, et al. Concentration dependence of the Faraday rotation in the Pr containing oxide glasses. J. Magn. Magn. Mater, 1998, 185(2): 207-212.

[8] MALAKHOVSKII A V, EDELMAN I S, RADZYNER Y, et al. Magnetic and magneto-optical properties of oxide glasses containing $\mathrm{Pr}^{3+}, \mathrm{Dy}^{3+}$ and $\mathrm{Nd}^{3+}$ ions. J. Magn. Magn. Mater., 2003, 263(1): $161-172$.

[9] NISHIBU S, NISHIO T, YONEZAWA S, et al. Preparation and magnetic properties of $\mathrm{TbF}_{3}$ containing oxide fluoride glasses. $J$. Fluorine Chem., 2006, 127(6): 821-823.

[10] HAYAKAWA T, NOGAMI M, NISHI N, et al. Faraday rotation effect of highly $\mathrm{Tb}_{2} \mathrm{O}_{3} / \mathrm{Dy}_{2} \mathrm{O}_{3}$-concentrated $\mathrm{B}_{2} \mathrm{O}_{3}-\mathrm{Ga}_{2} \mathrm{O}_{3}-\mathrm{SiO}_{2}-\mathrm{P}_{2} \mathrm{O}_{5}$ glasses. Chem. Mater., 2002, 14(8): 3223-3225.

[11] GOLIS E P, INGRAM A. Investigations of magneto-optic properties in $\mathrm{PbO}-\mathrm{Bi}_{2} \mathrm{O}_{3}-\mathrm{GeO}_{2}$ glass system. Journal of Physics: Conference Series, 2007, 79(1): 012003.

[12] OVCHARENKO N V, SMIRNOVA T V. High refractive index and magneto-optical glasses in the systems $\mathrm{TeO}_{2}-\mathrm{WO}_{3}-\mathrm{Bi}_{2} \mathrm{O}_{3}$ and $\mathrm{TeO}_{2}-\mathrm{WO}_{3}$-PbO. J. Non-Cryst. solids, 2001, 291(1): 121-126.

[13] RUAN Y, JARVIS R A, RODE A V, et al. Wavelength dispersion of Verdet constants in chalcogenide glasses for magneto-optical waveguide devices. Opt. Commun., 2005, 252(1): 39-45.

[14] WILliams P A, ROSE A H, DAY G W, et al. Temperature dependence of the Verdet constant in several diamagnetic glasses. Appl. Opt., 1991, 30(10): 1176-1178.

[15] DURCOK S, POLLERT E, SIMSA Z, et al. Growth of YIG and BiGdIG single crystals for magnetooptical applications. Mater. Chem. Phys., 1996, 45(2): 124-129.

[16] PARK M B, CHO N H. Structural and magnetic characteristics of yttrium iron garnet (YIG, Ce: YIG) films prepared by RF magnetron sputter techniques. J. Magn. Magn. Mater., 2001, 231(2): 253-264.

[17] SHIRAISHI K, NISHINO K, KAWAKAMI S, et al. Temperature-insensitive fiber Faraday rotator. Appl. Opt., 1985, 24(13): 1896-1897.

[18] CHEN Z, HANG Y, YANG L, et al. Fabrication and characterization of cerium-doped terbium gallium garnet with high magneto- optical properties. Opt. Lett., 2015, 40(5): 820-822. 
[19] CHEN Z, HANG Y, YANG L, et al. Great enhancement of Faraday effect by Pr doping terbium gallium garnet, a highly transparent VI-IR Faraday rotator. Mater. Lett., 2015, 145: 171-173.

[20] KHAZANOV E A, KULAGIN O V, YOSHIDA S, et al. Investigation of self-induced depolarization of laser radiation in terbium gallium garnet. IEEE J. Quantum Elect., 1999, 35(8): 1116-1122.

[21] VOITOVICH A V, KATIN E V, MUKHIN I B, et al. Wide-aperture Faraday isolator for kilowatt average radiation powers. Quantum Electron., 2007, 37(5): 471.

[22] CHANI V I, YOSHIKAWA A, MACHIDA H, et al. $(\mathrm{Tb}, \mathrm{Yb})_{3} \mathrm{Al}_{5} \mathrm{O}_{12}$ garnet: crystal-chemistry and fiber growth by micro-pulling-down technique. Mater. Sci. Eng. B, 2000, 75(1): 53-60.

[23] CHANI V I, YOSHIKAWA A, MACHIDA H, et al. Melt growth of $(\mathrm{Tb}, \mathrm{Lu})_{3} \mathrm{Al}_{5} \mathrm{O}_{12}$ mixed garnet fiber crystals. J. Cryst. Growth, 2000, 212(3): 469-475.

[24] GANSCHOW S, KLIMM D, EPELBAUM B M, et al. Growth conditions and composition of terbium aluminum garnet single crystals grown by the micro pulling down technique. J. Cryst. Growth, 2001, 225(2): 454-457.

[25] GEHO M, SEKIJIMA T, FUJII T, et al. Growth of terbium aluminum garnet $\left(\mathrm{Tb}_{3} \mathrm{Al}_{5} \mathrm{O}_{12} ; \mathrm{TAG}\right)$ single crystals by the hybrid laser floating zone machine. J. Cryst. Growth, 2004, 267(1): 188-193.

[26] SANGHERA J, KIM W, VILlalobOS G, et al. Ceramic laser materials: past and present. Opt. Mater., 2013, 35(4): 693-699.

[27] LI J, ZHOU J, PAN Y, et al. Solid-state reactive sintering and optical characteristics of transparent Er: YAG laser ceramics. J. Am. Ceram. Soc., 2012, 95(3): 1029-1032.

[28] LEE S H, KOCHAWATTANA S, MESSING G L, et al. Solid-state reactive sintering of transparent polycrystalline Nd: YAG ceramics. J. Am. Ceram. Soc., 2006, 89(6): 1945-1950.

[29] KOPYLOV Y L, KRAVCHENKO V B, BAGAYEV S N, et al. Development of $\mathrm{Nd}^{3+}: \mathrm{Y}_{3} \mathrm{Al}_{5} \mathrm{O}_{12}$ laser ceramics by high-pressure colloidal slip-casting (HPCSC) method. Opt. Mater., 2009, 31(5): 707-710.

[30] IKESUE A, KINOSHITA T, KAMATA K, et al. Fabrication and optical properties of high - performance polycrystalline Nd: YAG ceramics for solid-state lasers. J. Am. Ceram. Soc., 1995, 78(4): 1033-1040.

[31] 潘裕柏, 李 江, 姜本学. 先进光功能透明陶瓷. 北京: 科学出 版社, 2013.

[32] ATATURE M, DREISER J, BADOLATO A, et al. Observation of Faraday rotation from a single confined spin. Nat. Phys., 2007, 3(2): 101-106.

[33] YASUHARA R, TOKITA S, KAWANAKA J, et al. Cryogenic temperature characteristics of Verdet constant on terbium gallium garnet ceramics. Opt. Express, 2007, 15(18): 11255-11261.

[34] ZEPER W B, GREIDANUS F, CARCIA P F, et al. Perpendicular magnetic anisotropy and magneto-optical Kerr effect of vapordeposited Co/Pt-layered structures. J. Appl. Phys., 1989, 65(12): 4971-4975.

[35] CEBOLLADA A, WELLER D, STICHT J, et al. Enhanced magnetooptical Kerr effect in spontaneously ordered FePt alloys: quantitative agreement between theory and experiment. Phys. Rev.B, 1994, 50(5): 3419.

[36] ZHUANG N, SONG C, GUO L, et al. Growth of terbium gallium garnet (TGG) magneto-optic crystals by edge-defined film-fed growth method. J. Cryst. Growth, 2013, 381: 27-32.

[37] KHAZANOV E A. Investigation of Faraday isolator and Faraday Mirror Designs for Multi-kilowatt Power Lasers[C]//High-Power Lasers and Applications. International Society for Optics and Photonics, 2003: 115-126.

[38] KAGAN M A, KHAZANOV E A. Thermally induced birefrin- gence in Faraday devices made from terbium gallium garnetpolycrystalline ceramics. Appl. Opt., 2004, 43(32): 6030-6039.

[39] YASUHARA R, SNETKOV I, STAROBOR A, et al. Terbium gallium garnet ceramic Faraday rotator for high-power laser application. Opt. Lett., 2014, 39(5): 1145-1148.

[40] SNETKOV I L, YASUHARA R, STAROBOR A V, et al. TGG ceramics based Faraday isolator with external compensation of thermally induced depolarization. Opt. Express, 2014, 22(4): 4144-4151.

[41] LIN H, ZHOU S, TENG H. Synthesis of $\mathrm{Tb}_{3} \mathrm{Al}_{5} \mathrm{O}_{12}$ (TAG) transparent ceramics for potential magneto-optical applications. Opt. Mater, 2011, 33(11): 1833-1836.

[42] CHEN C, YI X, ZHANG S, et al. Vacuum sintering of $\mathrm{Tb}_{3} \mathrm{Al}_{5} \mathrm{O}_{12}$ transparent ceramics with combined $\mathrm{TEOS}+\mathrm{MgO}$ sintering aids. Ceram. Int., 2015, 41(10): 12823-12827.

[43] DAI J W, SNETKOV I L, PALASHOV O V, et al. Fabrication, microstructure and magneto-optical properties of $\mathrm{Tb}_{3} \mathrm{Al}_{5} \mathrm{O}_{12}$ transparent ceramics. Opt. Mater., 2016, 62: 205-210.

[44] 戴佳卫, 李 江, 潘裕柏, 等. 一种新型的制备琙铝石榴石基纳 米粉体及磁光透明陶瓷的方法. 专利, 201611239053.4, 2016$12-29$.

[45] 戴佳卫, 尹 瑞, 李 江, 等. 一种非水基凝胶注模成型制备铽铝 石榴石基磁光透明陶瓷的方法. 专利, 201610554361.X, 2016$07-15$.

[46] CHEN C, NI Y, ZHOU S, et al. Preparation of $\left(\mathrm{Tb}_{0.8} \mathrm{Y}_{0.2}\right)_{3} \mathrm{Al}_{5} \mathrm{O}_{12}$ transparent ceramic as novel magneto-optical isolator material. Chin. Opt. Lett., 2013, 11(2): 021601.

[47] CHEN C, ZHOU S, LIN H, et al. Fabrication and performance optimization of the magneto-optical $\left(\mathrm{Tb}_{1-x} \mathrm{R}_{x}\right)_{3} \mathrm{Al}_{5} \mathrm{O}_{12} \quad(\mathrm{R}=\mathrm{Y}, \mathrm{Ce})$ transparent ceramics. Appl. Phys. Lett., 2012, 101(13): 131908.

[48] FURUSE H, YASUHARA R, HIRAGA K, et al. High Verdet constant of Ti-doped terbium aluminum garnet (TAG) ceramics. Opt. Mater. Express, 2016, 6(1): 191-196.

[49] STAROBOR A, ZHELEZNOV D, PALASHOV O, et al. Study of the properties and prospects of Ce: TAG and TGG magnetooptical ceramics for optical isolators for lasers with high average power. Opt. Mater. Express, 2014, 4(10): 2127-2132.

[50] ZHELEZNOV D, STAROBOR A, PALASHOV O, et al. High-power Faraday isolators based on TAG ceramics. Opt. Express, 2014, 22(3): 2578-2583.

[51] ZHELEZNOV D, STAROBOR A, PALASHOV O, et al. Improving characteristics of Faraday isolators based on TAG ceramics by cerium doping. Opt. Lett., 2014, 39(7): 2183-2186.

[52] YASUHARA R, FURUSE H. Thermally induced depolarization in TGG ceramics. Opt. Lett., 2013, 38(10): 1751-1753.

[53] YASUHARA R, NOZAWA H, YANAGITANI T, et al. Temperature dependence of thermo-optic effects of single-crystal and ceramic TGG. Opt. Express, 2013, 21(25): 31443-31452.

[54] YASUHARA R, SNETKOV I, STAROBOR A, et al. Terbium gallium garnet ceramic-based Faraday isolator with compensation of thermally induced depolarization for high-energy pulsed lasers with kilowatt average power. Appl. Phys. Lett., 2014, 105(24): 241104.

[55] SNETKOV I L, PERMIN D A, BALABANOV S S, et al. Wavelength dependence of Verdet constant of $\mathrm{Tb}^{3+}: \mathrm{Y}_{2} \mathrm{O}_{3}$ ceramics. Appl. Phys. Lett., 2016, 108(16): 161905.

[56] FURUSE H, YASUHARA R. Magneto-optical characteristics of holmium oxide $\left(\mathrm{Ho}_{2} \mathrm{O}_{3}\right)$ ceramics. Opt. Mater. Express, 2017, 7(3): 827-833.

[57] MORALES J R, AMOS N, KHIZROEV S, et al. Magneto-optical Faraday effect in nanocrystalline oxides. J. Appl. Phys., 2011, 109(9): 093110. 